\title{
A Monte Carlo study of random surface field effect on layering transitions
}

\author{
H. Ez-Zahraouy*, L. Bahmad, and A. Benyoussef \\ Laboratoire de Magnétisme et de la Physique des Hautes Energies \\ Université Mohammed V, Faculté des Sciences, Avenue Ibn Batouta, \\ Rabat B.P. 1014, Morocco
}

\begin{abstract}
The effect of a random surface field, within the bimodal distribution, on the layering transitions in a spin- $1 / 2$ Ising thin film is investigated, using Monte Carlo simulations. It is found that the layering transitions depend strongly on the concentration $p$ of the disorder of the surface magnetic field, for a fixed temperature, surface and external magnetic fields. Indeed, the critical concentration $p_{c}(k)$ at which the magnetisation of each layer $k$ changes the sign discontinuously, decreases for increasing the applied surface magnetic field, for fixed values of the temperature $T$ and the external magnetic field $H$. Moreover, the behaviour of the layer magnetisations as well as the distribution of positive and negative spins in each layer, are also established for specific values of $H_{s}, H, p$ and the temperature $T$.
\end{abstract}

Keywords: Monte Carlo; surface field; thin film; random field; Layering transitions.

(*) Corresponding author: ezahamid@fsr.ac.ma

\section{Introduction}

Several authors have studied the layering transitions of magnetic Ising systems, such as Pandit et al. [1], Pandit and Wortis [2], Nightingale et al. [3] and Ebner et al. [4-7]. Many experimental studies have motivated these theoretical works. Indeed, several methods have been used to study the layering transitions in Ising magnetic films. Benyoussef and Ez-Zahraouy have studied these layering transitions using a real space renormalization group [8], and transfer matrix methods [9]. Using the mean field theory, Hong [10], have found that depending on the values of the exchange integrals near the surface region, the film critical temperature may be lower, higher than, or equal to that of the bulk. Using the perturbative theory, Harris [11] have showed the existence of layering transitions at $T=0$ in the presence of a transverse magnetic field. The effect of finite size on such transitions has been studied, in a thin film confined between parallel plates or walls, by Nakanishi and Fisher [12] using mean field theory and by Bruno etal. [13] taking into account the capillary condensation effect. By applying 
Monte Carlo simulations on thin Ising films with competing walls, Binder et al. [14], found that occurring phase transitions belong to the universality class of the two-dimensional Ising model and found that the transition is shifted to a temperature just below the wetting transition of a semi-infinite fluid [15,16]. Hanke etal. [17] showed that symmetry breaking fields give rise to nontrivial and long-ranged order parameter profiles for critical systems such as fluids, alloys, or magnets confined to wedges. Much attention has been paid to the properties of layered structures consisting of alternating magnetic materials. The most commonly studied magnetic multilayers are those of ferromagnetic transition metal such as $C o$ or $N i$. Many experiments have shown that the magnetization enhancement exists in multilayered films consisting of magnetic layers. It was found that ferromagnetic coupling can exist between magnetic layers. From the theoretical point of view, great interest has been paid to spin wave excitations as well as critical phenomena [18-21]. The study of thin films is partly motivated by the development of new growth and characterisation techniques, but perhaps more so by the discovery of many exciting new properties, some quite unanticipated. These include, more recently, the discovery of enormous values of magnetoresistance in magnetic multilayers far exceeding those found in single layer films and the discovery of oscillatory interlayer coupling in transition metal multilayers. These experimental studies have motivated much theoretical work. However these developments are applied to a large extent powered by "materials engineering" and the ability to control and understand the growth of thin layers only a few atoms thick. However, Many experimental studies have shown that the magnetisation enhancement exists in multilayered films consisting of magnetic layers. On the other hand, we have shown in one of our earlier works [22] the existence of layering transitions under the effect of a variable surface coupling. Moreover, for an Ising film with a wedge, we found in Ref. [23] the intra-layering transitions under the geometry effect consisting on the existence a wedge. When the film is subject to a random transverse magnetic field [24], we found the layer-by-layer transitions when increasing the concentration $p$ above a critical value $p_{c}(k)$ for each layer $k$. The aim of this work is to study the effect a random surface magnetic field on layering transitions of an Ising thin film, using Monte Carlo simulations. The paper is organised as follows. In section 2, we describe the model and the method used: Monte Carlo (MC) simulations. In section 3 we present results and discussions.

\section{Model and Monte Carlo simulations}

We are studying a magnetic thin film formed with $N=4$ layers coupled ferromagnetically. Each layer is a square of dimension $N_{x} \times N_{y}=64 \times 64$ spins. $N_{x}$ and $N_{y}$ stand for the number of spins in the $x$ and $y$ directions, respectively.

A preliminary study showed that, when performing Monte Carlo simulations under the Metropolis algorithm, we note that the relevant calculated quantities did not change appreciably for small film thickness: $N=3, N=4, N=5$ and $N=8$ layers; and when varying the number of spins of each layer from $N_{x}=N_{y}=32$ to 128. Taking into account the above considerations, in all the following we will give numerical results for a film thickness $N=4$ layers, and $N_{x}=N_{y}=64$ spins for each layer.

A surface magnetic field $H_{s}$ is acting only on spins of the surface $k=1$.

The Hamiltonian governing the system is given by

$$
\mathcal{H}=-J \sum_{<i, j>} S_{i} S_{j}-\sum_{i} H_{i} S_{i}
$$


where, $S_{l}(l=i, j)=-1,+1$ are the spin variables and the interaction between different spins is assumed to be constant. The total magnetic field $H_{i}$ applied on each site $i$ of the layer $k$, is distributed according to:

$$
H_{i}=\left\{\begin{array}{cll}
H+H_{s i} & \text { for all sites } \mathrm{i} \epsilon & \text { surface } \mathrm{k}=1 \\
H & \text { for all sites i } \epsilon & \text { layers } \mathrm{k}=2,3,4 .
\end{array}\right.
$$

$H$ is an external magnetic field, and $H_{s i}$ is the surface magnetic field distributed according to a bimodal law:

$$
\mathcal{P}\left(H_{s i}\right)=p \delta\left(H_{s}-H_{s i}\right)+(1-p) \delta\left(H_{s i}\right),
$$

so that the surface magnetic field is:

$$
H_{s i}=\left\{\begin{array}{cc}
H_{s} & \text { with the probability } \mathrm{p} \\
0 & \text { with the probability 1-p }
\end{array}\right.
$$

where $0 \leq p \leq 1$. For $p=1, H_{s}$ is acting on all sites of the surface; whereas $p=0$ corresponds to a situation with no surface magnetic field: $H_{s}=0$.

\section{Results and discussion}

We present in Fig. 1 the ground state phase diagram in the plane $\left(H, H_{s}\right)$ for a film with $N=4$ layers, with different line transitions and configurations. For $H_{s} \geq H_{s c}=N /(N-1)$, the system transits from the configuration $O^{N}$ to the configuration $1 O^{N-1}$ at the line $H=1-H_{s}$; and the configuration $1 O^{N-1}$ transits to $1^{N}$ at a constant magnetic field $H=-1 /(N-1)$. Whereas the transition from the configuration $O^{N}$ to $1^{N}$ is located at $H=-H_{s} / N$, provided that the surface magnetic field is positive and kept less than its critical value: $H_{s} \leq H_{s c}$. It is worth to note that all these configurations are located at negative values of the external magnetic field $H$. In this work, the temperature fluctuations will not be taken into account. Hence, the temperature will be fixed at the value $T=3.75$ in all the following. In order to outline the effect of the surface magnetic field concentration, we plot in Fig. 2 the phase diagram of the studied system in the plane $(H, p)$, for $H_{s}=1.5$. We show the existence of two critical probabilities: $p_{L}^{S}$ and $p_{L}^{F}$. The former is the layering concentration corresponding to the transition of the surface $k=1$, and located at about $\approx 0.40$. The surface line transition is terminated by the endpoint $C_{1}$ located at the critical concentration $p_{c}(1) \approx 0.60$. The latter is the film transition located at approximately $\approx 0.55$ as it is shown in the inset of Fig. 2 . It is worth to note that the internal layers of the film transit at different lines with different endpoints. Indeed, the second layer $k=2$ transits at line terminated by the endpoint $C_{2}$ located at $\approx 0.94$, the third layer corresponds to the line with the endpoint $C_{3}$ located at $\approx 0.92$, whereas the last layer $k=4$ at the line with the endpoint $C_{4}$ corresponding to the critical concentration $\approx 0.88$.

These critical probabilities depend strongly on the surface magnetic field values, at a fixed external magnetic field as it illustrated by Fig. 3. When increasing $H_{s}$, the critical concentration of the surface $p_{c}(1)$ as well as for internal layers $k=2,3,4, p_{c}(k)$ decreases when increasing $H_{s}$ more and more. In all cases the critical concentration of the surface $p_{c}(1)$ is always found to be smaller than $p_{c}(k), k=2,3,4$ of the other layers. This is due to the fact that the surface 
transits, in all cases, before the other layers. This is outlined in Fig. 4, for a small concentration $p=0.30$ of $H_{s}$ for a fixed surface magnetic field $H_{s}=1.5$. To complete this study, we give in Figs. $5 a, 5 b$ the distribution of spins of the surface before and after the transition shown in Fig. 4 , for $H_{s}=1.5$ and $p=0.30$. Fig. $5 a$ shows the map of negative spins for $H=-0.20$ before the transition, whereas Fig. $5 b$ corresponds to positive spins for $H=-0.05$ after the transition located at an external magnetic field $H \approx-0.08$. This magnetic field value depends not only on the concentration $p$ but also on the surface magnetic field $H_{s}$; and decreases when increasing the concentration $p$. Indeed, the random distribution of $H_{s}$ is responsible on the formation of small and randomly distributed islands of negative and positive spins present in the surface and also in the deeper layers $k=2,3,4$. When $p \rightarrow 1.0$ the negative spins, or positive ones, are assembled on lager islands in a given layer and the randomness of $H_{s}$ is lost.

\section{Conclusion}

We have studied the effect of a random surface magnetic field, within the bimodal distribution, on the layering transitions of a spin-1/2 Ising thin film formed with $N=4$ layers, using Monte Carlo simulations. We showed that the layering transitions depend strongly on the concentration $\mathrm{p}$ of the surface magnetic field, for a fixed surface and external magnetic fields. Moreover, the critical concentration $p_{c}(k)$ of each layer $k$ decreases with increasing the applied surface magnetic field, for a fixed value of the external magnetic field. In order to complete this study, we have investigated the magnetisation behaviour for each layer as a function of the external magnetic field for fixed values of $T, H_{s}$, and the concentration $p$. We have also shown the existence of islands of negative spins surrounded by aggregation of positive spins.

\section{References}

[1] R. Pandit, M. Schick and M. Wortis, Phys. Rev. B 26, 8115 (1982).

[2] R. Pandit and M. Wortis, Phys. Rev. B 25, 3226 (1982).

[3] M. P. Nightingale, W. F. Saam and M. Schick, Phys. Rev. B 30,3830 (1984).

[4] C. Ebner, C. Rottman and M. Wortis, Phys. Rev. B 28,4186 (1983).

[5] C. Ebner and W. F. Saam, Phys. Rev. Lett. 58,587 (1987).

[6] C. Ebner and W. F. Saam, Phys. Rev. B 35,1822 (1987).

[7] C. Ebner, W. F. Saam and A. K. Sen, Phys. Rev. B 32,1558 (1987).

[8] A. Benyoussef and H. Ez-Zahraouy, Physica A, 206, 196 (1994).

[9] A. Benyoussef and H. Ez-Zahraouy, J. Phys. I France 4, 393 (1994).

[10] Q. Hong Phys. Rev. B 41, 9621 (1990); ibid, Phys. Rev. B 46, 3207 (1992).

[11] A. B. Harris, C. Micheletti and J. Yeomans, J. Stat. Phys. 84, 323 (1996)

[12] H. Nakanishi and M. E. Fisher, J. Chem. Phys. 78,3279 (1983) 
[13] P. S. Swain and A. O. Parry, Eur. Phys. J. B 4, 459 (1998); E. Bruno, U. Marini, B. Marconi and R. Evans, Physica A 141A, 187 (1987)

[14] K. Binder, D. P. Landau and A. M. Ferrenberg, Phys. Rev. Lett. 74, 298 (1995)

[15] K. Binder, D. P. Landau and A. M. Ferrenberg, Phys. Rev. E 51, 2823 (1995)

[16] M. Bengrine, A. Benyoussef, H. Ez-Zahraouy and F. Mhirech, Physica A 268, 149 (1999)

[17] A. Hanke, M. Krech, F. Schlesener and S. Dietrich, Phys. Rev. E 60, 5163 (1999)

[18] S. Dietrich and M. Schick, Phys. Rev B 31,4718 (1985)

[19] S. J. Kennedy and S. J. Walker, Phys. Rev. B 30,1498 (1984)

[20] P. Wagner and K. Binder, Surf. Sci. 175,421 (1986)

[21] K. Binder and D. P. Landau, Phys. Rev. B 37, 1745 (1988)

[22] L. Bahmad, A. Benyoussef, and H. Ez-Zahraouy, Surf. Sci. 536, 114 (2003)

[23] L. Bahmad, A. Benyoussef, and H. Ez-Zahraouy, Phys. Rev. E 66, 056117 (2002)

[24] L. Bahmad, A. Benyoussef, and H. Ez-Zahraouy, Chin. J. Phys. 40, 537 (2002)

\section{Figure Captions}

Figure 1.:

The ground state phase diagram in the plane $\left(H, H_{s}\right)$ for a film with $N=4$ layers, with different line transitions and configurations.

\section{Figure 2:}

Phase diagram in the plane $(H, p)$ for a fixed temperature $T=3.75$ and a surface magnetic field value $H_{s}=1.5$, showing the layering transition probability of the surface $p_{L}^{S}$ as well as the endpoints $C_{k}, k=1,2,3,4$. Inset, the layering transition probability of the film $p_{L}^{F}$ and the layering probability of the remaining layers.

\section{Figure 3:}

Critical probabilities $p_{c}(k)$ for each layer, as a function of the surface magnetic field $H_{s}$ for an external magnetic field $H=-0.20$.

\section{Figure 4:}

Magnetisation profiles as a function of $H$ for different layers $k=1,2,3,4$ of the film, for $H_{s}=1.5$ and a concentration value $p=0.30$. The number accompanying each curve is the layer order.

\section{Figure 5:}

Distribution of spins of the surface $k=1$ before and after the transition for a film with $N=4$ layers, $T=3.75, H_{s}=1.5$ and $p=0.30$ : (a) negative spins for $H=-0.20$ before the transition, (b) positive spins for $H=-0.05$ after the transition. 
Fig. 1

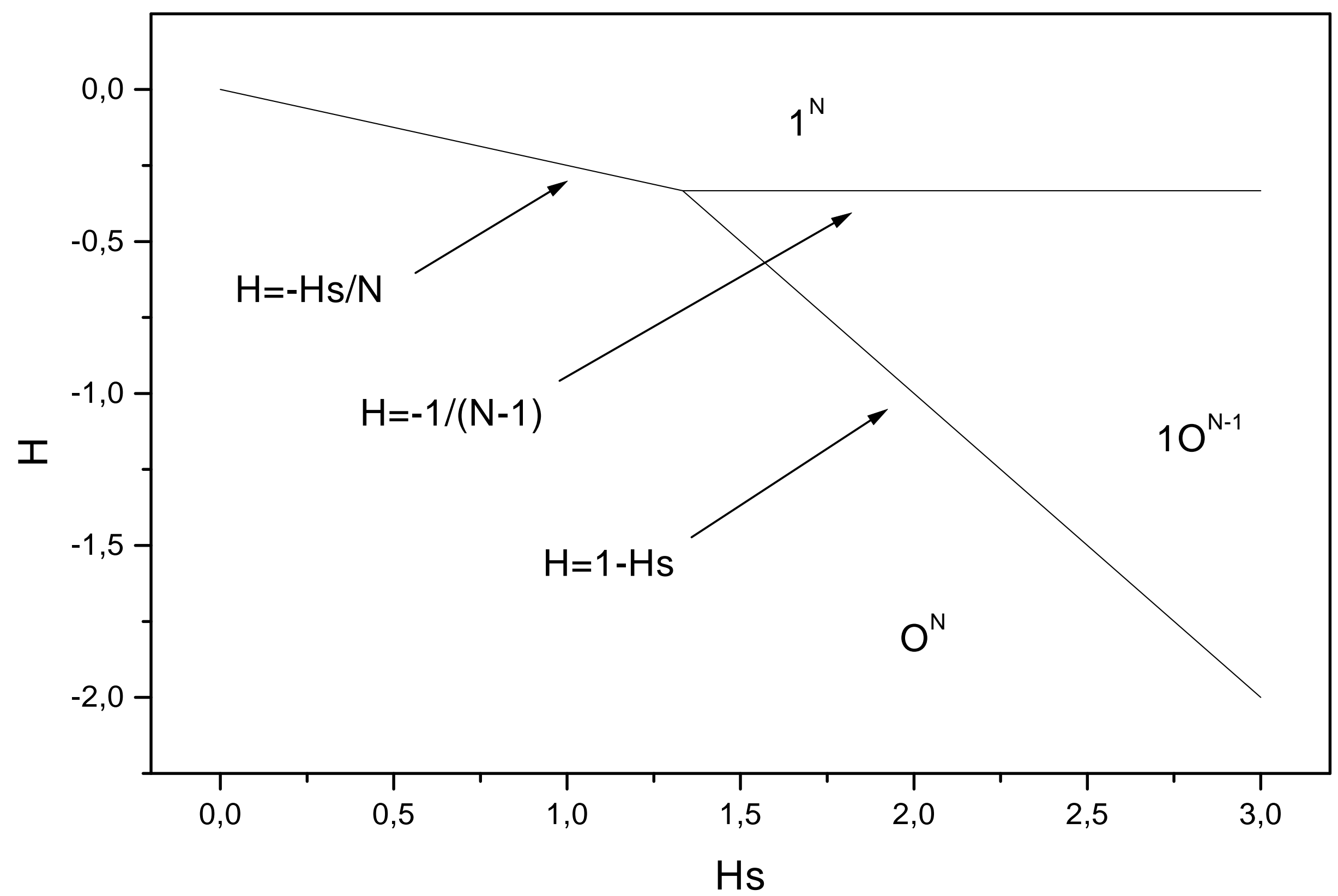


Fig. 2

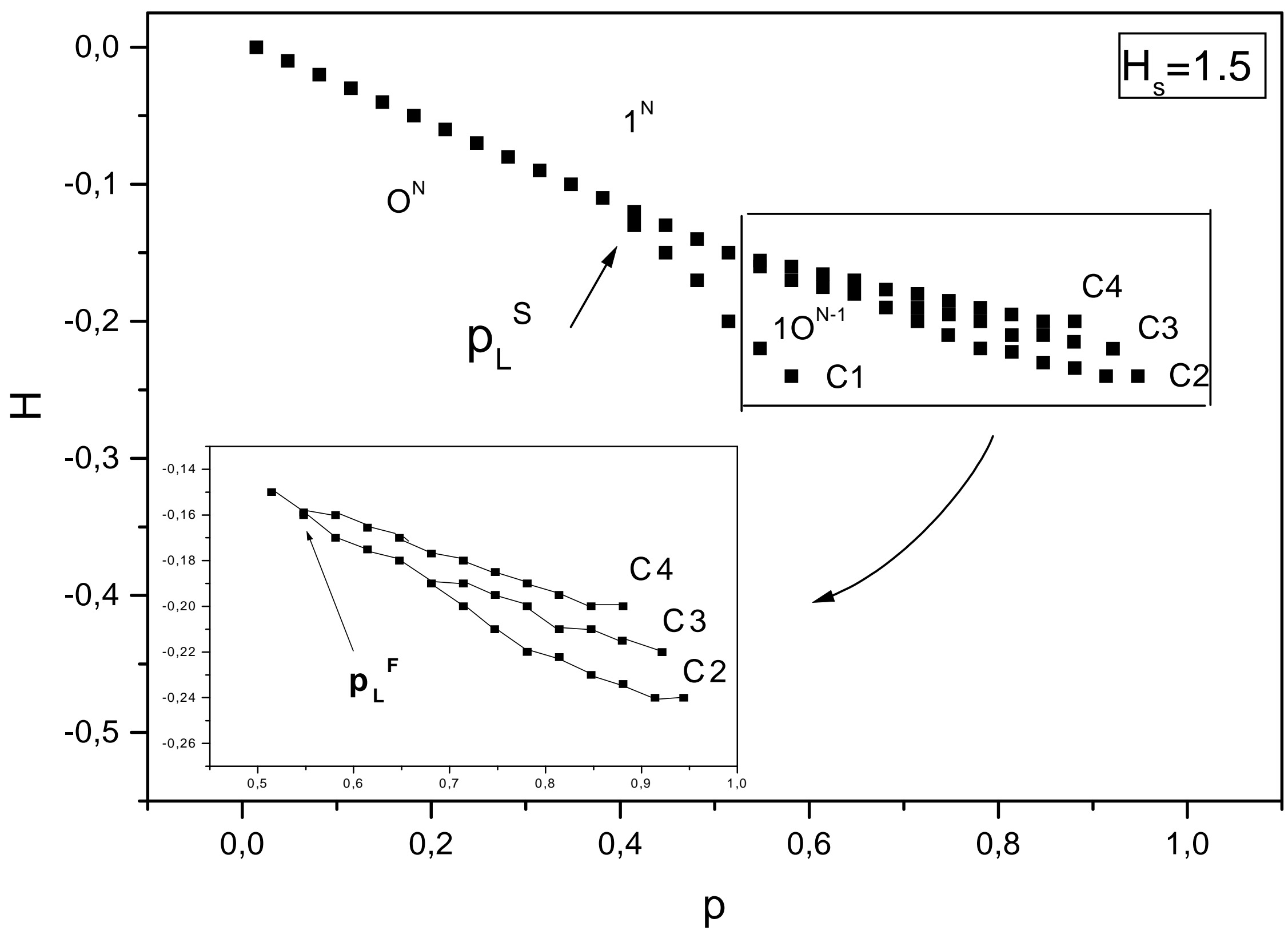


Fig. 3

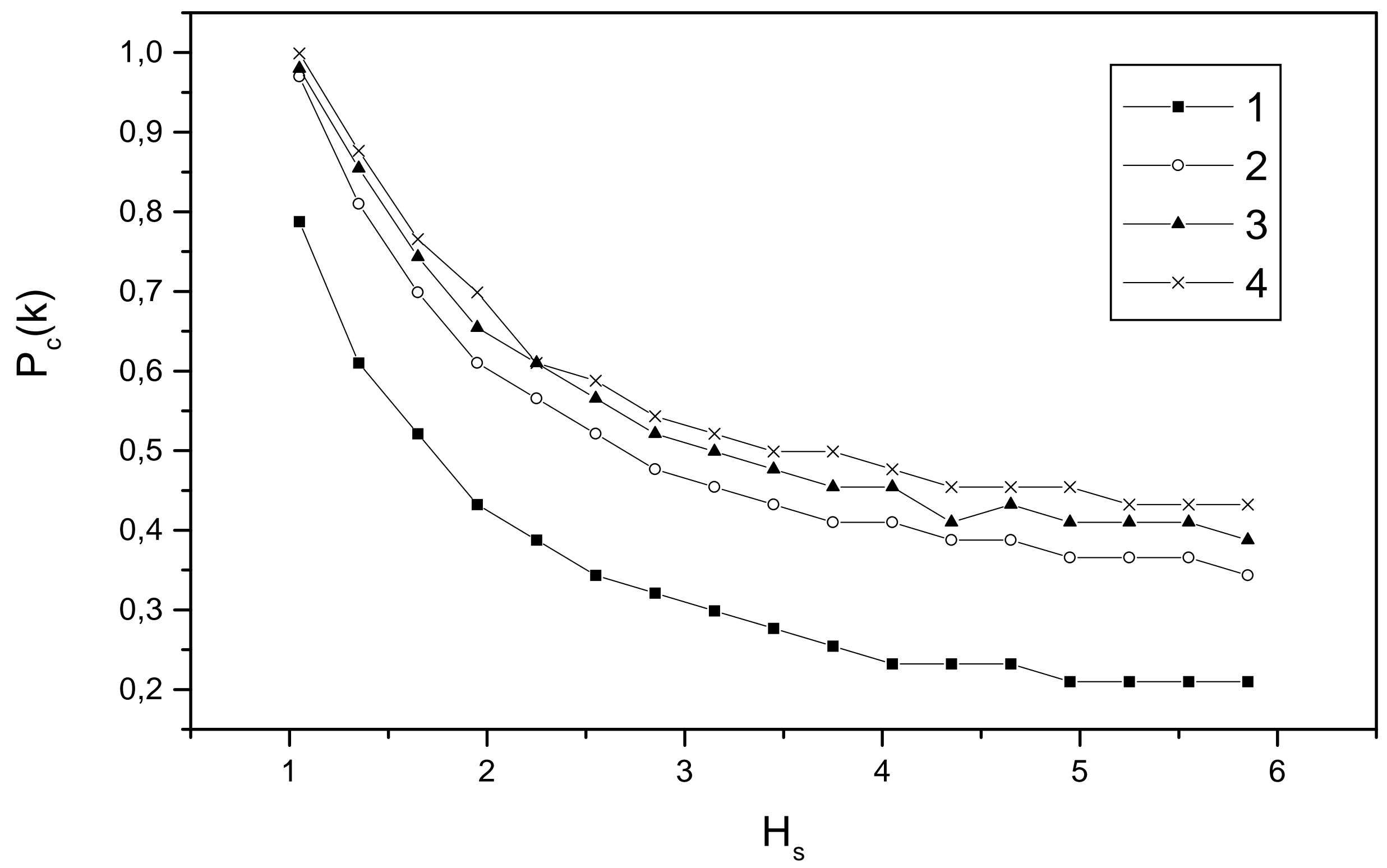




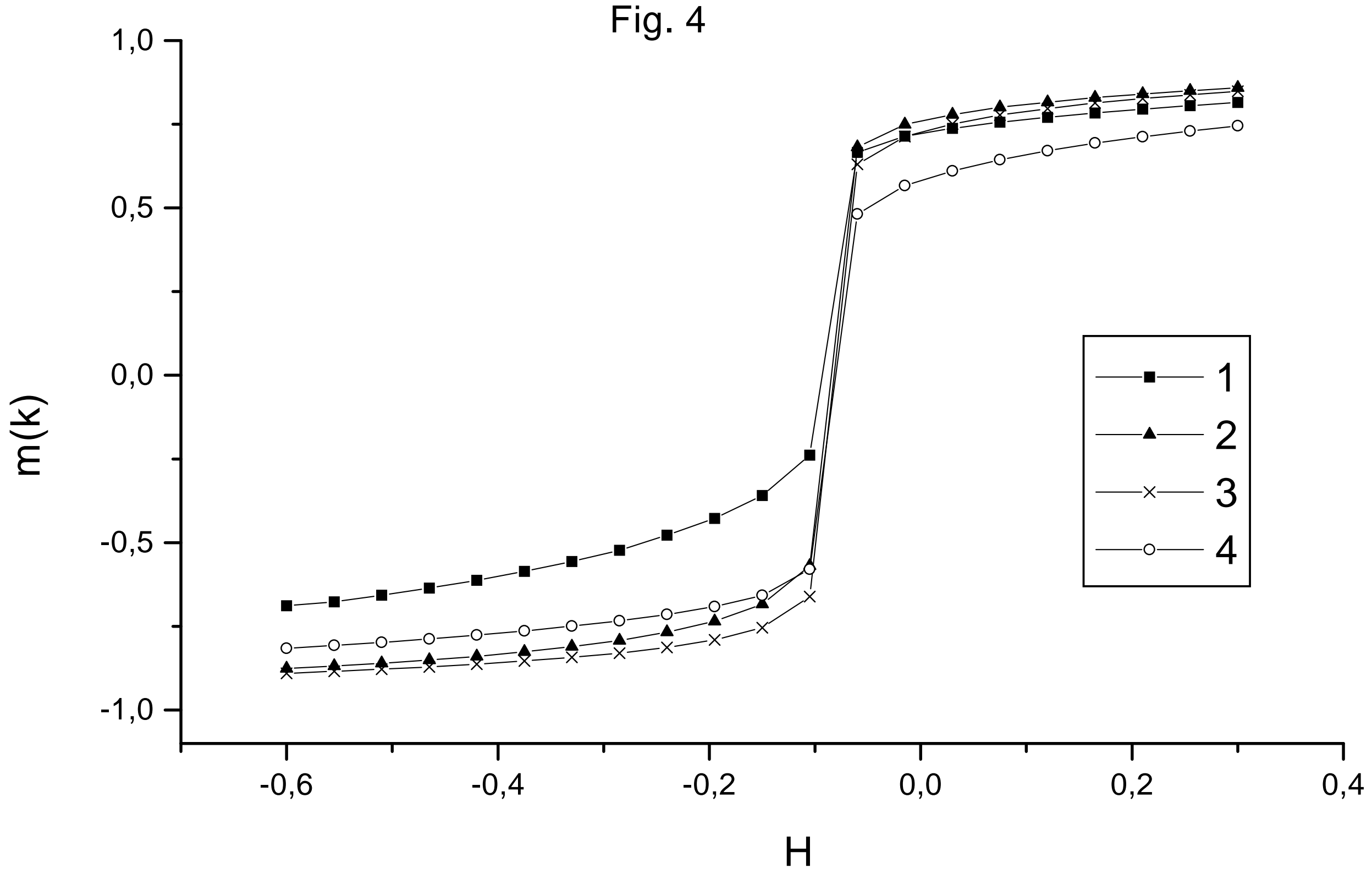




\section{Fig. 5b}

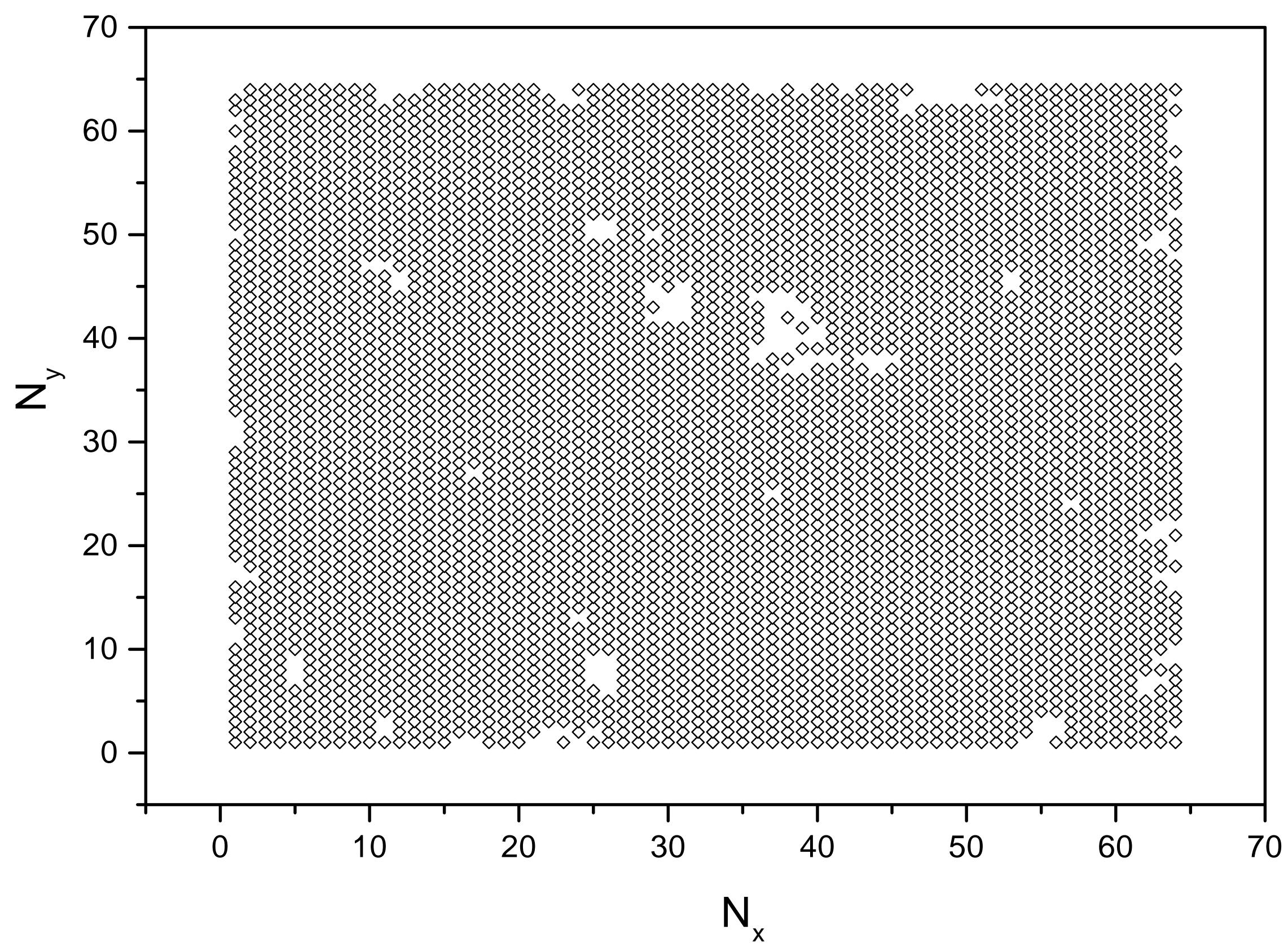




\section{Fig. 5a}

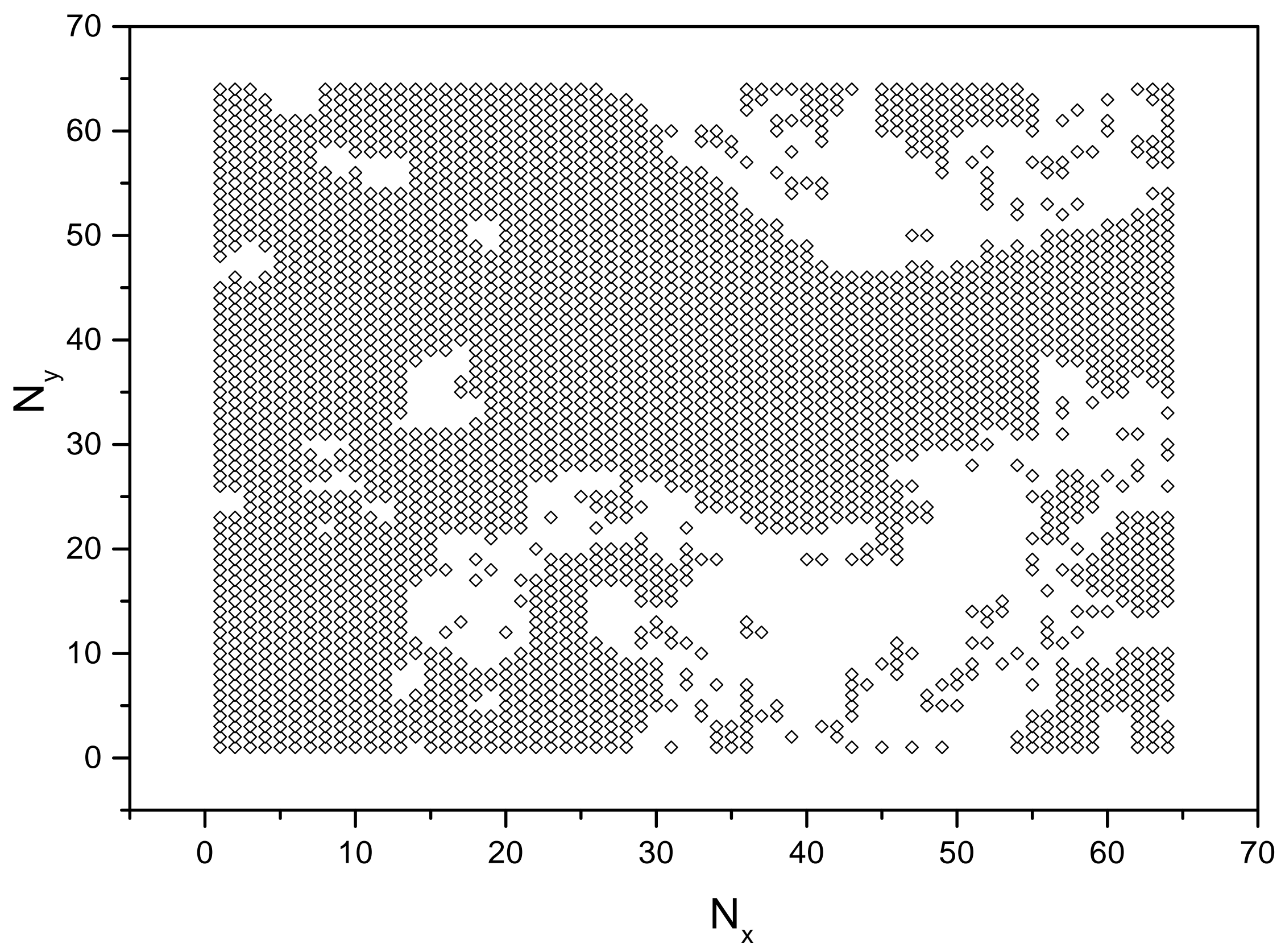

